\title{
TUMOR ODONTOGÉNICO QUERATOQUÍSTICO INFECTADO: CARACTERÍSTICAS IMAGENOLÓGICAS Y SEGUIMIENTO: REPORTE DE CASO
}

\section{INFECTED ODONTOGENIC KERATOQUISTIC TUMOR: IMAGE CHARACTERISTICS AND FOLLOW-UP: CASE REPORT}

\author{
Paucar-Oyola Jean ${ }^{1 *}$, Ruiz-García Villma ${ }^{2}$ \\ ${ }^{1}$ Residente del segundo año de Radiología Oral y Maxilofacial - Facultad de Estomatología Universidad Peruana Cayetano \\ Heredia. Perú \\ 2 Docente de imagenología de la FE Roberto Beltrán Universidad Peruana Cayetano Heredia. Perú \\ *jean.paucar.o@upch.pe
}

\begin{abstract}
Resumen
El Tumor Odontogénico Queratoquístico (TOQ) es una neoplasia odontogénica benigna de los maxilares. Suele presentarse durante la segunda y tercera década de vida con un ligero predominio hacia el sexo masculino, su ubicación más común es el cuerpo o rama mandibular. El TOQ no suele presentar sintomatología, pero en algunos casos pueden presentar dolor y aumento de volumen de la zona afectada. Radiográficamente se presenta como una imagen radiolúcida de límites definidos, con bordes festoneados y corticalizados y cuando presenta una infección secundaria estas características pueden verse alteradas. Esta entidad tiene una alta recurrencia debido a que después del tratamiento pueden quedar quistes satélites en el interior del lecho quirúrgico. El presente reporte muestra las características imagenológicas de un TOQ con una infección secundaria de larga data y su persistencia luego del tratamiento quirúrgico.
\end{abstract}

Palabras clave: quistes odontogénicos, infecciones bacterianas, diagnóstico por imagen (DeCS) .

\begin{abstract}
Keratocystic odontogenic tumor (KCOT) is a benign odontogenic neoplasm of the jaw. Usually presents during the second and third decade of life with a slight predominance towards males, its most common locations are the body or ramus mandibular. KCOT doesn't usually present symptomatology, but in some cases it does produce pain and increase of volume of the affected area. It presents as a radiolucent image with clear limits, scalloped and cortical borders. If a secondary infection is present these characteristics may be altered. This entity has a high recurrence if cysts satellites remain inside the surgical bed. The present report shows the image characteristics of a TOQ with a long no-treated secondary infection and its persistence after surgical treatment
\end{abstract}

Key words: odontogenic cysts, bacterial infections, imaging diagnostic (MeSH).

\section{INTRODUCCIÓN}

El Tumor Odontogénico Queratoquístico (TOQ) es una neoplasia benigna de los maxilares con un potencial agresivo e infiltrativo. Fue descrito por primera vez por Philipsen en 1956 como Queratoquiste Odontogénico y reclasificado por la Organización Mundial de la Salud (OMS) en el año 2005 como Tumor Odontogénico Queratoquístico por su naturaleza neoplásica. ${ }^{1,2}$ Se puede originar por restos de la lámina dental, extensión de las células del epitelio basal o degeneración de las células del retículo estrellado y se postulan 3 causas para su crecimiento: aumento de replicación de células basales, aumento de la presión osmótica o liberación de factores de reabsorción ósea. ${ }^{3}$ Suele presentarse durante la segunda y tercera década de vida, con un ligero predominio al sexo masculino, su ubicación más común es el cuerpo o rama mandibular; y la presentación en maxilar superior con compromiso del seno maxilar es rara. ${ }^{4}$ Dentro de sus características clínicas el TOQ no suele presentar sintomatología, en 
muchos casos su diagnóstico es un hallazgo radiográfico; en algunos casos pueden presentar dolor y aumento de volumen, expansión y drenaje de la zona afectada. ${ }^{5}$ Radiográficamente se presenta como una imagen radiolúcida unilocular o multilocular de límites definidos y bordes corticalizados, de crecimiento agresivo que puede desplazar piezas dentarias y adelgazar las corticales. Cuando presenta una infección secundaria estas características se pueden verse alteradas., ${ }^{6,7}$ La presentación múltiple del TOQ es rara y generalmente está asociada junto con otras anomalías al síndrome de Gorlin Goltz. ${ }^{8}$ Con respecto a sus características tomográficas se presenta como una imagen isodensa bien delimitada con un contorno festoneado sin expansión cortical. ${ }^{9}$ Dentro del pronóstico, es importante señalar que el TOQ tiene una alta tasa de recurrencia debido a que después del tratamiento quedan en el interior del lecho quirúrgico pequeños quistes satélites o fragmentos de epitelio. ${ }^{10}$ Otro factor importante en el TOQ es la asociación a una infección secundaria ya que puede producir dolor e inflamación además de ocasionar cambios en las imágenes radiográficas. El objetivo de este reporte es presentar las características imagenológicas de un TOQ con una infección secundaria de larga data y su persistencia luego del tratamiento quirúrgico.

\section{PRESENTACIÓN DEL CASO}

Paciente masculino de 29 años de edad acudió al servicio de Cirugía Oral y Maxilofacial de la Clínica Estomatológica Central de la Universidad Peruana Cayetano Heredia debido a dolor, tumefacción y trismus a nivel del ángulo mandibular del lado derecho, con un tiempo de enfermedad de 1 año y 7 meses, de inicio insidioso y curso progresivo. Paciente refirió que empezó a sentir dolor en la región submandibular del lado derecho, por lo cual acudió a una clínica particular y fue derivado al área de Oncología y hospitalizado por 2 días. Al ser evaluado por este servicio le diagnostican "Adenitis ganglionar", por lo que fue medicado con antibióticos y antiinflamatorios no esteroideos sin disminuir los síntomas. En el año 2014 le indican una radiografía panorámica donde le detectan un proceso infeccioso asociado a un tumor mandibular en el ángulo mandibular derecho, razón por la cual es sometido a un abordaje extraoral para drenar el proceso infeccioso. En el examen extraoral el paciente presenta una fascie no característica, aumento de volumen en la región maseterina y submandibular a nivel del ángulo mandibular derecho de consistencia firme, bordes difusos y con un leve aumento de la temperatura en la zona. En el examen intraoral la apertura bucal está limitada, mucosas orales poco hidratadas y el fondo de surco vestibular del cuarto cuadrante estaba conservada y sin presencia de secreciones, no presenta linfoadenopatías palpables ni movilidad dentaria. Como plan de trabajo para el diagnóstico se indicó una radiografía panorámica, en la cual se observó una imagen radiolúcida de límites definidos y bordes parcialmente corticalizados en ángulo y rama mandibular del lado derecho, multilocular, de

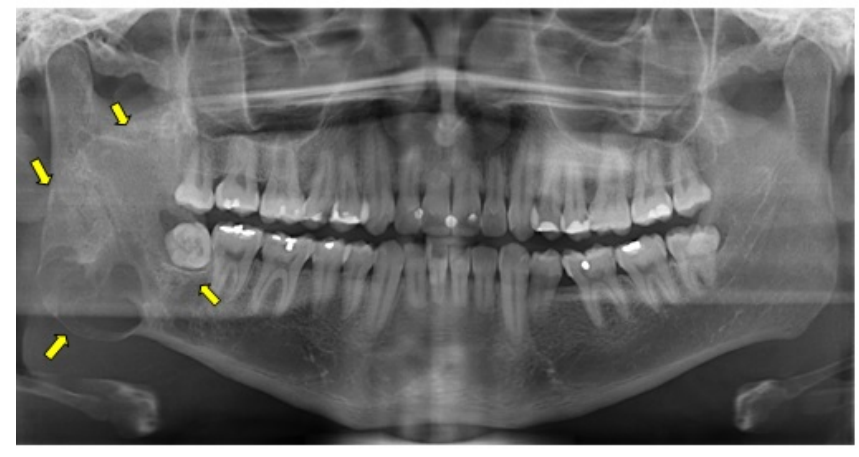

Fig. 1. Radiografía Panorámica: Imagen radiolúcida en ángulo y rama mandibular derecha.

forma irregular que produce la expansión y adelgazamiento del borde anterior de la rama, ángulo y basal mandibular. La lesión respeta las corticales del conducto dentario inferior y está en relación a la pieza 48 la cual se encuentra en torsión bucolingual. A la evaluación de las demás estructuras hay un engrosamiento de la mucosa del seno maxilar izquierdo y una reabsorción radicular de las piezas 11, 21, 31, 32, 41, 42 así como múltiples restauraciones coronarias. (Fig. 1) Posteriormente se le indicó una Tomografia Espiral Multicorte (TEM), en la cual se observa una imagen isodensa de límites definidos en ángulo y rama mandibular del lado derecho que produce el engrosamiento de la rama mandibular del lado derecho, adelgazamiento, perforación de la tabla ósea lingual y expansión caudal del ángulo mandibular (Fig. 2 y 3). El músculo masetero y el tejido celular subcutáneo presentan un cambio en la densidad producto de la infección sobreagregada y (Fig. 4) a la evaluación de Unidades Hounsfield (UH) dió distintos valores dentro de la lesión desde 13 hasta 181 (Fig.5). Se llegó como conclusión imagenológica de Tumor Odontogénico infectado, a descartar TOQ infectado. Se le realizó la extracción de la pieza 48 y una biopsia cuyo resultado fue "fragmentos de queratoquiste". Con este diagnóstico fue sometido al tratamiento de marsupialización desde el cual se ha mantenido en controles periódicos. Se realizó la marsupialización y en los controles radiográficos 9 meses después del tratamiento la lesión muestra mejor definición de sus límites y bordes parcialmente corticalizados pero con persistencia (Fig.6). Al año 6 meses en la radiografía panorámica se observa un aumento en las dimensiones de la lesión (Fig.7).

\section{DISCUSIÓN}

En el año 2005 la OMS reclasificó al Queratoquiste como una neoplasia intraósea benigna renombrándola como Tumor Odontogénico Queratoquístico (TOQ) por su naturaleza neoplásica. ${ }^{11} \mathrm{Se}$ origina de los restos de la lámina dental y representa el $2 \%$ de los tumores odontogénicos benignos. ${ }^{12}$ Pueden ocurrir a cualquier edad pero básicamente se diag- 


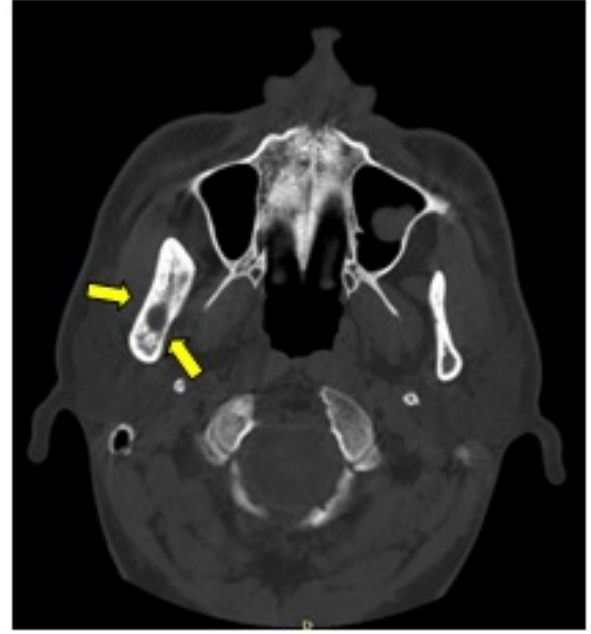

Fig. 2. TEM corte axial en ventana para tejidos duros. Engrosamiento de la rama mandibular del lado derecho.

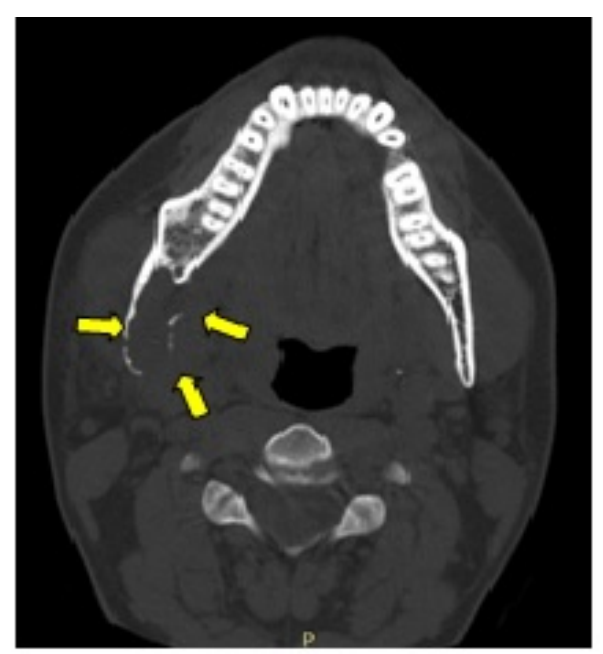

Fig. 3. TEM corte axial en ventana para tejidos duros. Se evidencia la expansión y perforación de la tabla ósea lingual.

nostican durante la segunda y tercera década de vida. En su mayoría se presentan en el maxilar inferior. Radiográficamente el TOQ se presenta en cuerpo y rama mandibular como una imagen radiolúcida unilocular o multilocular, de límites definidos y bordes corticalizados. A pesar del crecimiento agresivo presenta ligera expansión de las tablas óseas, puede desplazar piezas dentarias y adelgazar las corticales anatómicas, ${ }^{13}$ dichos signos son apreciables también en la TEM. ${ }^{14}$ La TEM es un examen auxiliar imagenólogico de gran utilidad que nos brinda la posibilidad de evaluar tridimensionalmente las estructuras del macizo facial y visualizar tejidos blandos y óseos en relación a las estructuras adyacentes. En la TEM podemos evaluar las UH las cuales son en realidad valores de pixel que representan el coeficiente de atenuación de un

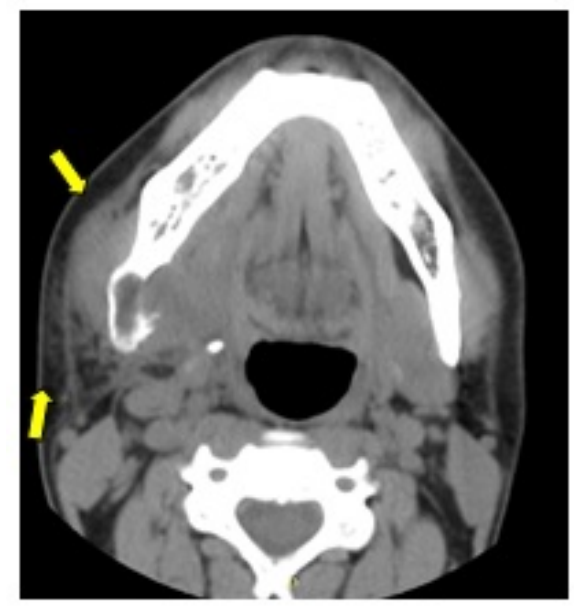

Fig. 4. TEM corte axial ventana para tejidos blandos. Cambio de la densidad del tejido celular subcutáneo y del musculo masetero.
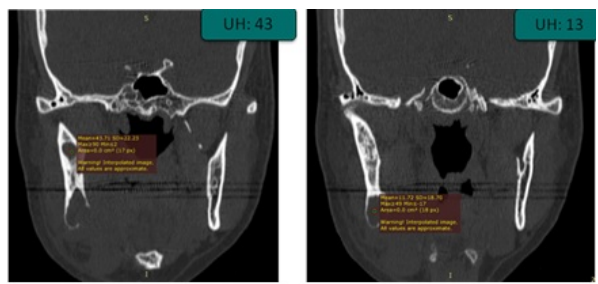

Fig. 5. TEM corte coronal en ventana para tejidos duros. Medición de las UH en distintos sectores de la lesión.

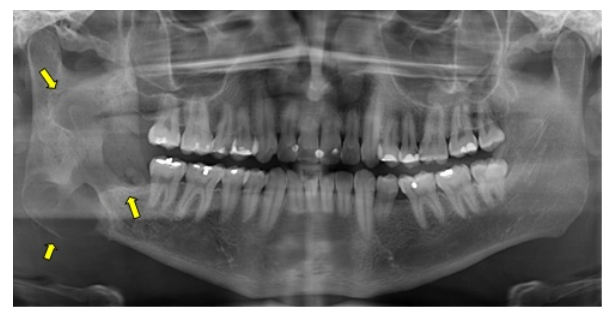

Fig. 6. Radiografía panorámica 9 meses después del tratamiento de marsupialización.

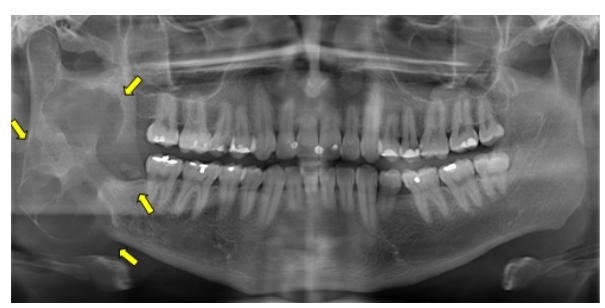

Fig. 7. Radiografía panorámica 1 año 6 meses después del tratamiento de marsupialización. La lesión muestra aumento de tamaño. 
elemento de volumen que está representado por una escala donde el 0 corresponde al agua, -1000 al aire y +1000 el hueso compacto. Por lo tanto, el valor de estas nos ayudarán en el diagnóstico. ${ }^{15}$ Otro aporte de la TEM es que se puede usar la administración de un medio de contraste que permiten resaltar y opacificar estructuras anatómicas normales y patológicas. También evalúan la perfusión y permiten diferenciar las interfases o densidades entre los distintos tejidos con fines médicos (diagnósticos o terapéuticos). ${ }^{16} \mathrm{El}$ TOQ está entre 70 a 80 UH por el contenido de queratina, cuando hay valores más altos puede deberse a que hay un proceso de desnaturalización de la proteína, por el contrario valores más bajos puede deberse a un proceso de infección sobreagregada. ${ }^{17}$ En este caso se midieron las UH en 5 sitios de la lesión: $43 \mathrm{UH}$ en rama mandibular, borde anterior de la rama y centro de la lesión, 13 UH en el ángulo y 181 UH en cuerpo mandibular, confirmando que hay un proceso de infección sobreagregada, y además que hay un cambio en la estructura ósea cerca del cuerpo mandibular ya que el valor normal del hueso trabecular es de $400 \mathrm{UH} .{ }^{17}$ En ocasiones la patología puede tener un proceso de infección sobreagregada que producirá cambios en su apariencia imagenológica: hay pérdida de las corticales óseas que delimitan la lesión, los límites se vuelven poco definidos, la conformación interna con aspecto nuboso y disminución de los valores de UH. ${ }^{17,18}$

En este caso hubo una pérdida de las corticales en la lesión, los límites eran parcialmente definidos en rama y cuerpo mandibular, la estructura interna presentaba una densidad nubosa y los valores UH descendieron a 43. Dadas estas características se podía afirmar la presencia de un proceso de infección sobreagregado. Bodner y cols. ${ }^{19}$ hicieron un estudio para evaluar las características de formación de hueso tras el tratamiento de marsupialización bajo 4 parámetros y 4 categorías descritos por Kawai y cols. ${ }^{20}$ en una investigación anterior. El primero fue el contorno de la lesión (sin cambios, ligeramente modificado, parcialmente reducido y reducido), el segundo fue la distancia entre la lesión y las estructuras adyacentes (disminuido, sin cambios, ligeramente mayor y aumentado). La tercera integridad del canal mandibular y el hueso cortical (sin cambios, ligeramente remodelada, remodelada y completamente remodelado) y la cuarta características de formación ósea (sin cambios, vidrio esmerilado, espiculado y trabeculado). Como resultado encontraron que la regeneración ósea es más rápida con la enucleación en comparación con la marsupialización. En este caso no hubo ningún signo de reparación ni cambios en los 4 parámetros descritos según Bodner y cols. ${ }^{19}$ lo que lleva a la reflexión que hubo varios aspectos sobre los cuales la reparación ósea, luego del tratamiento de marsupialización, no se dio bajo los parámetros mencionados. Además, hay factores asociados a esta etapa después del tratamiento como son el cuidado propio del paciente de la herida operatoria y de la continuidad 11 de sus controles periódicos tanto clínicos como imagenológicos. La recurrencia del TOQ puede estar relacionada en varios aspectos como el periodo de seguimiento, el tipo de tratamiento, la técnica adecuada del cirujano, la extensión de la lesión y la zona en que se produjo la misma. Con esto se han formulado varias hipótesis como la eliminación incompleta del revestimiento epitelial y la persistencia de quistes satélites que no han sido retirados en el procedimiento quirúrgico. ${ }^{19}$ En conclusión las características imagenológicas de un TOQ pueden verse alteradas si hay un proceso de infección secundaria generando dificultad en el diagnóstico y afectar el éxito del tratamiento. La persistencia de la lesión puede deberse a varios factores como el seguimiento, técnica y extensión de la lesión lo que va impedir el proceso de reparación ósea esperado como se relata en el presente caso.

Conflicto de intereses y financiamiento Los autores declaran no tener conflicto de intereses, haber cumplido con los requisitos de autoría y haber autofinanciado este artículo.

\section{Referencias}

1 Marcotullio D, Iannella G, Zelli M, Marinelli C, Magliulo G. Rare and massive odontogenic parakeratotic cyst treated by endoscopic sinus surgery: a case report. J Med Case Rep. 2014;8(293): 1-4.

2 Vázquez D, Gandini P, Ramírez M, Ibero J, Carbajal E. Keratocystic odontogenic tumor: Radiographic findings and surgical management of a clinical case. Av Odontoestomatol 2012; 28(5): 249-54.

3 Robles P, Roa I. Keratocystic odontogenic tumor: Clinicopathological aspects and treatment. J Oral Res. 2014; 3(4):249-56.

4 Lacarbonara M, Marzo G, Lacarbonara V, Monaco A, Capogreco M. Presentation of a keratocystic odontogenic tumor with agenesis: a case report. J Med Case Rep. 2014;8 (126): 1-7.

5 Zachariades N, Papanicolaou S, Triantafyllou D. Odontogenic keratocysts: review of the literature and report of sixteen cases. J Oral Maxillofac Surg 1985; 43(3):177-82.

6 White S, Pharoah M. Oral radiology principles and interpretation.6ta ed. Missouri: Mosby Elsevier ; 2009.

7 Langlais R, Langland O, Nortje C. Diagnostic Imaging of the jaws. 1a ed. Malvern. William y Wilkins. 1995.

8 Ba K, Li X, Wang H, Y Liu, Zheng G, Yang Z et al. Correlation between imaging features and epithelial cell proliferation in keratocystic odontogenic tumor. Dentomaxillofac Rad. 2010 (39): 368-74.

9 Larheim TA, Westesson P. Maxillofacial Imaging. 1a ed. Berlin. Springer. 2006.

10 Zhao Y, Liu B, Cheng G, Wang S, Wang Y. Recurrent keratocystic odontogenic tumours: report of 19 cases. Dentomaxillofac Rad. 2012; 41(2): 96-102.

11 Philipsen HP, Reichart PA: Classification of odontogenic tumours: a historical review. J Oral Pathol Med. 2006; (35):525-29. 
12 Kuroyanagi N, Sakuma H, Miyabe S, Machida J, Kaetsu A, Yokoi $\mathrm{M}$ et al. Prognostic factors for keratocystic odontogenic tumor (odontogenic keratocyst): analysis of clinicopathologic and immunohistochemical findings in cysts treated by enucleation. J Oral Pathol Med. 2009;(38):386-92.

13 Kimura T, Ohba S, Yoshimura H, Fujita S, Imamura Y, Kitagawa $\mathrm{Y}$ et al. Keratocystic odontogenic tumor arising at the mandibular ramus with an impacted tooth: a case report and mimic lesions. Cranio. 2014; 1(1): 1-6.

14 Theodorou S, Theodorou D, Sartoris D. Imaging characteristics of neoplasms and other lesions of the jaw . Odontogenic tumors and tumorlike lesions. Clin Imag. 2007;31(2):114-9.

15 Dellán A, Villaroel M, Hernandez-Andara A. Aplicación de las unidades Hounsfield en tomografia computarizada como herramienta diagnóstica de las lesiones intraóseas del complejo maxilo mandibular: estudio clínico de diagnostico. Rev Odontol Univ Cid Sao Paulo. 2015; 27(2): 100-11.

16 Cifuentes D , Aguirre G. Pautas para la aplicación controlada de medios de contraste endovasculares. Rev Fac Med. 2014;22(1):78-83.

17 Davoodi P, Rezael-Soufi L, Jazaeri M,Langaroodi A, Hoselni S. Submandibular abscess due to an infected keratocystic odontogenic tumor asocciated with simultaneous ocurrence of a traumatic bone cyst: a rare case report. J Contemp Dent Pract. 2013; 14(1): 133-6.

18 De Azambuja S, Bastos A, Couto P, Motohiro O, Ditzel V, Westphalen F. Spontaneus eruption of a canine after marsupialization of an infected dentigerous cyst. Am J Orthod Dentofac. 2008; 137 (5): 690 -3.

19 Bodner L, Bar-Ziv J. Characteristics of bone formation following marsupialization of jaw cyst. Dentomaxillofac Rad. 1998; 27: 166 - 71.

20 Kawai T, Murakami S, Hiranuma H, Sakuda M. Healing after removal of benign and tumors of the jaws. Oral surg Oral Med O. 1995; 79: 17-25.

Recibido: 20 de Agosto de 2016

Aceptado: 27 de Agosto de 2016 
